\title{
Public Field Trial of a Multi-RAT (60 GHz 5G/LTE/WiFi) Mobile Network
}

\author{
Matthias Steeg, Nathan J. Gomes, Adrian A. Juarez, Michał Kościesza, Mason Lange, Yigal Leiba, \\ Hiroshi Mano, Hiroshi Murata, Michał Szczęsny, and Andreas Stöhr
}

\begin{abstract}
A public field trial showcasing an operational multiRadio Access Technology (RAT) mobile network that was implemented in one of the largest shopping mall in Warsaw, Poland. The network supports novel $60 \mathrm{GHz} 5 \mathrm{G}$ mobile access as well as legacy LTE and WiFi services All mobile access services of the network are interconnected via optical fiber to the data centers of a mobile network operator and an internet service provider. Fronthauling for the $60 \mathrm{GHz} 5 \mathrm{G}$ hotspot radio access unit (RAU) and for LTE is realized by analog Radio-over-Fiber (RoF) via a fiber-optic distributed antenna system (DAS). The $60 \mathrm{GHz} 5 \mathrm{G}$ radio access units (RAUs) for the enhanced mobile broadband (eMBB) use case and the WiFi access point (AP) are both backhauled via optical Gigabit Ethernet. The $60 \mathrm{GHz}$ RAUs for the eMBB and hotspot use case feature 2D beam-switching and 1D beam-steering, respectively. Inter-RAT switching between the different mobile services with seamless user experience is achieved using a Mobile IP system with Fast Initial Link Setup (FILS).
\end{abstract}

\section{INTRODUCTION}

$\mathrm{T}$ HE advent of the fifth generation of mobile communications $(5 \mathrm{G})$ is promising to change the way people use the Internet and interact with it. Two figures that are often highlighted to illustrate this are a 1000 -fold increase in mobile data traffic as well as a reduction of latency below the $1 \mathrm{~ms}$ threshold [1]-[3]. The year 2018 plays an important role in evaluating how far we have come towards reaching this goal technically; also, major standardization documents for key markets are expected to be released in 2018. Moreover, major stakeholders have announced field trials to show the status of their pre-commercial solutions [4]. In this context, we report on the field trial of the European-Japanese RAPID5G consortium that successfully demonstrated a $5 \mathrm{G}$ network to the public in one of the largest shopping mall in Poland. The second field trial of the RAPID5G consortium in a football stadium in Osaka, Japan, will be reported elsewhere. The aim of the RAPID5G field trial in Poland was to study and demonstrate a heterogeneous network supporting novel $60 \mathrm{GHz} 5 \mathrm{G}$ radio

This work supported by the Horizon2020 EU-JP research project RAPID5G (www.rapid-5g.eu or www.rapid-5g.jp) under the grant no.643297.

M. Steeg, M. Lange and A. Stöhr are with the Center for Semiconductor and Optoelectronics, University of Duisburg-Essen, 47057 Duisburg, Germany (email: andreas.stoehr@uni-due.de). N. Gomes is with the School of Engineering and Digital Arts, University of Kent, Canterbury, UK (e-mail: N.J.Gomes@kent.ac.uk). A. A. Juarez is with Corning Optical Communications GmbH \& Co. KG, 10117 Berlin, Germany (e-mail: service for high data rates supporting the enhanced mobile broadband (eMBB) use case family but also legacy LTE and WiFi networks for ubiquitous coverage. The eMBB use case is seen as a trailblazer technology to establish the $5 \mathrm{G}$ platform, as it addresses the key necessities, which are already imminent in todays' mobile communications such as high data rates and dense user environments [1],[4]. RAPID5G has chosen a large shopping mall in Europe for its field trial as it represents a natural dense user environment in which multiple different services and ubiquitous coverage is a must [5]. In order to meet the high data rate demands expected for the eMBB use case in $5 \mathrm{G}$, we have developed novel radio access technologies (RATs) operating in the $60 \mathrm{GHz}$ millimeter-wave frequency range [2],[6]. One is a $60 \mathrm{GHz}$ RAU for the $5 \mathrm{G}$ hotspot use case supporting multiple Gbit/s uncompressed video streaming at mm-wave frequencies. The $60 \mathrm{GHz}$ hotspot RAU has a fiber input and is able to generate multiple steerable beams simultaneously. Therefore, it supports dense user areas up to the extreme beam-per-mobile-user use case. Analog RoF is utilized for fronthauling the $60 \mathrm{GHz}$ hotspot RAU [6]-[8]. Analog RoF can provide very dense cell allocations with flexible reconfiguration as well as low latencies [7]. Furthermore, they provide the necessary fronthaul bandwidth and even support inexpensive receiver architectures based on envelope detectors [8]. The second $60 \mathrm{GHz} 5 \mathrm{G} 60$ radio access technology developed in RAPID5G is based upon a novel $60 \mathrm{GHz}$ SiGe transceiver chip in combination with a lens-assisted 2D beam switching antenna. This $60 \mathrm{GHz}$ RAU features full-duplex TDD communication using a commercial Gigabit-Ethernet (GbE) interface. The integrated beam switching solution allows for user mobility, wide angle coverage and supports horizontal handover between various $60 \mathrm{GHz}$ cells [2]. In addition to the $60 \mathrm{GHz}$ mobile access also LTE and WiFi service was provided by the network. All radio access technologies were interconnected to the data center of a mobile network operator (MNO) and the data center of an internet service provider using a fiber-optic distributed antenna system (DAS) that was also

juareza2@corning.com). M. Kościesza was with Exatel SA. M. Szczęsny is with Exatel SA, 04-164 Warsaw, Poland (e-mail: michal.szczesny@exatel.pl). Y. Leiba is with Siklu Communication Ltd., Petach Tikva 49517, Israel (e-mail: yigal@siklu.com). H. Mano is with Koden Techno Info Co.,Ltd., Tokyo 1070061, Japan (e-mail: mano@koden-ti.com). H. Murata was with the Graduate School of Engineering Science, Osaka University, Osaka, Japan and joined the Graduate School Engineering, Mie University, Mie, Japan, in 2018 (e-mail: murata@elec.mie-u.ac.jp). 


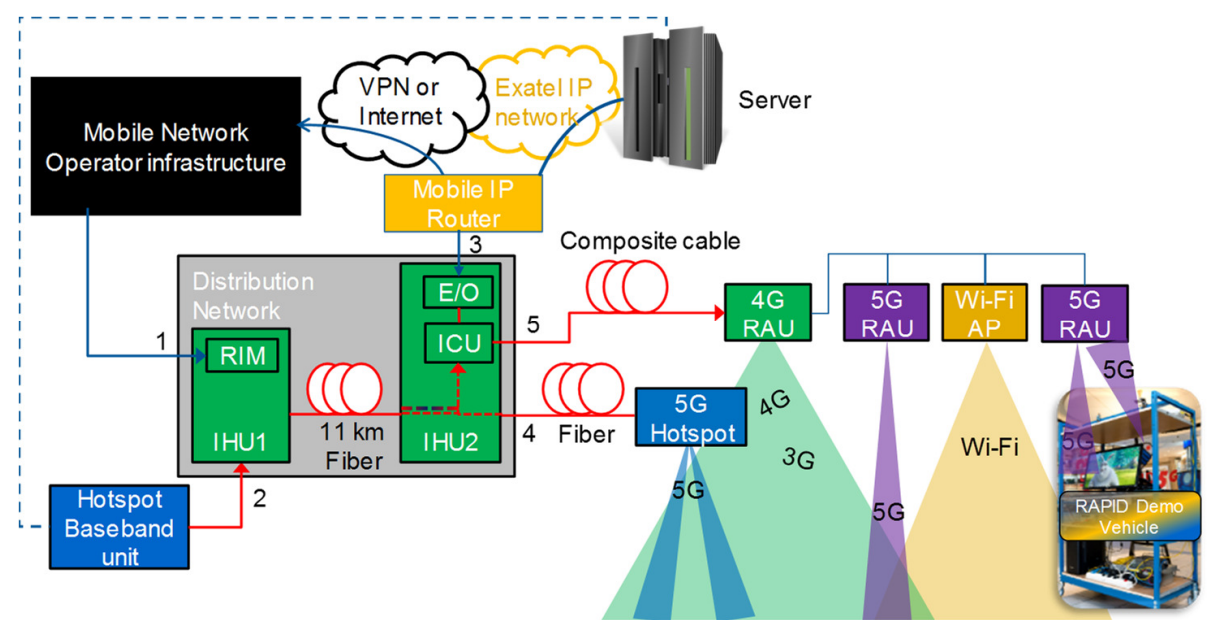

Fig. 1. Block diagram of the RAPID5G network implemented in the shopping mall in Warsaw, Poland. The color coding of the different radio access units (RAUs) and access points (APs) refers to the different radio access technologies employed in the heterogeneous wireless access network. The distribution network spanning between the two integrated headend units (IHUs), and their modular components from the radio interface module (RIM) to the interconnect unit (ICU) is described in detail in section IV. A. Distributed Antenna System.

developed within RAPID5G. In order to provide low-latency seamless vertical handover between the different access technologies, a Mobile IP system was developed and implemented [8]-[10]. The RAPID5G Mobile IP system includes a fast initial link setup (FILS) protocol to decrease the overall switching time [11]. By using Mobile IP and FILS, we successfully demonstrated virtually seamless vertical handover between $5 \mathrm{G}$, LTE and WiFi. It was for example demonstrated that active applications (e.g. video streaming) running on the mobile terminal (MT) are not interrupted when changing the physical layer RAT between novel $60 \mathrm{GHz}$ access and legacy LTE or WiFi.

\section{NETWORK SETUP}

The overall goal of the RAPID5G project was to deliver broadband $5 \mathrm{G}$ services within the $60 \mathrm{GHz}$ band in dense user areas. Thus RAPID5G targeted the eMBB and hotspot use cases. Since the existence of $5 \mathrm{G}$ radio will not lead to the immediate termination of legacy wireless services, future $5 \mathrm{G}$ services should coexist and cooperate with the dominant legacy wireless access services to provide the best user experience [1],[3]-[5]. For this reason, RAPID5G aimed at developing and implementing a heterogeneous wireless access network with a seamless switching technology for vertical and horizontal handoff based upon Mobile IP and FILS. The Mobile IP home agent is a core part of the radio resource management in the RAPID5G network and was implemented in the field trial demonstrations.

For a realistic network setup, all data for the field trial needs to be hosted outside the shopping mall. This means that all RAUs of the multi RAT network (WiFi, LTE, $60 \mathrm{GHz} 5 \mathrm{G}$ ) must be connected to the Mobile IP home agent server as well as to the data servers of the MNO and internet provider. To achieve this, a distributed antenna system (DAS) was developed by Corning, which interconnects the various RAUs in the public demonstration area of the shopping mall with the outside world as illustrated in Fig. 1. The server, hosting the data to be accessed in the field trial and the mobile IP server are both located at Exatel's data centers in Warsaw and are connected to the shopping mall over optical fiber via the IP network. The LTE base station of a Polish MNO was installed in one of Exatel's data centers and remoted via optical fiber to the data center of the MNO which is also located in the city center of Warsaw.

\section{MOBILE IP FOR UBIQUitous COVERAGE}

\section{A. Mobile IP Implementation}

The RAPID5G network operates within the environment formed by the following three different radio access technologies: novel 5G $60 \mathrm{GHz}$, LTE and WiFi. In such a heterogeneous network, the mobile terminal (MT) must have the capability to move and dynamically attach itself to the most suitable link, in terms of current radio conditions. In order to achieve this kind of heterogeneous mobility, a Mobile IP system has been developed and implemented in the RAPID5G shopping mall field trial.

RAPID5G's Mobile IP system is based on a protocol and architecture defined in IETF's RFC 5944 [8]. The overall aim of this architecture is to provide network layer roaming capability between IP subsets. Thanks to that, the mobile node can move from one LAN segment to another, as long as its IP address stays unchanged. This is possible because in the Mobile IP system, the mobile node is assigned two types of IP addresses. The first is the home address $-\mathrm{a}$ virtual address that remains the same regardless of the RAT it is attached to. The second is the care-of address, which is associated with the physical network interface. Unlike the home address, the careof address changes whenever the mobile node roams to a different LAN segment. In such a configuration, the Mobile IP protocol is responsible for registering and de-registering, the logical association between the currently assigned care-of address and the fixed home address in the Home Agent server. Therefore, the Home Agent is always "aware" of the IP location of the mobile node and can act as an anchor point for incoming and outgoing traffic by creating Generic Routing Encapsulation (GRE) tunnels. 
Diagnostic panel

\begin{tabular}{|l|c|}
\hline 5G U1 status & connected \\
\hline 5G U1 quality & $20 \%$ \\
\hline 5G U2 status & connected \\
\hline 5G U2 quality & $20 \%$ \\
\hline WiFi signal quality & $94 \%$ \\
\hline WiFi level & $-44 \mathrm{dBm}$ \\
\hline Modem RF type & LTE \\
\hline Modem signal quality & $53 \%$ \\
\hline Modem level & $-66 \mathrm{dBm}$ \\
\hline
\end{tabular}

Highest throughput RF

Download $817172 \mathrm{~kb} / \mathrm{s}$

Upload $2990 \mathrm{~kb} / \mathrm{s}$

RTD time $5.7 \mathrm{~ms}$

\begin{tabular}{|l|l} 
MIP process & running
\end{tabular}

\begin{tabular}{l|c|c|}
\hline MIP socket state $\quad$ connected \\
\hline
\end{tabular}

\begin{tabular}{|l|r|r|}
\hline MIP state & registered
\end{tabular}

MIP active RF $\quad 5 \mathrm{G}$

Handover Auto 5G Wifi LTE

Fig. 2. Diagnostic panel of the radio management software for monitoring and controlling the network performance in the shopping mall field trial.

Due to its versatile capabilities, Mobile IP was incorporated into the CDMA2000 network and nowadays plays a key role in the recent 3GPP LTE standards suite [10].

In the RAPID5G field trial, the mobile terminal (MT) was equipped with three physical network interfaces: Ethernet (directly attached to the $5 \mathrm{G} 60 \mathrm{GHz}$ RAT adapter), $802.11 \mathrm{WiFi}$ and an LTE modem via USB connection. The installed software stack consisted of the Mobile IP Agent responsible for Mobile IP registrations and GRE tunneling establishment, and the Handover Agent (HA) whose role was to gather network and radio condition parameters and make handover decisions based on that data. The RAPID5G terminal frequently monitors the RSSI (received signal strength indicator) level, signal power level, data rate, round trip delay and other performance parameters. Thus, when the RAPID5G terminal was moving and in case a RSSI hysteresis threshold is exceeded, the Handover Agent requests the Mobile IP Agent to switch transmission to a more suitable radio link. To achieve an efficient handover, we have implemented a make-before-break mechanism and optimized the RSSI threshold levels for stable operation. On the request, the Mobile IP Agent re-registered the home address of the mobile terminal with an updated care-of address for the other radio link. An internal IP routing table would be reconfigured, so that the incoming IP stream was redirected to the GRE tunnel established through the new radio link. In consequence, the mobile terminal was able to move and seamlessly switch between various radio access technologies without breaking the service, which was presented during the field trial via an uninterrupted video stream.

\section{B. Mobile IP Extensions for Fast Initial Link Setup and policy-based routing}

In addition to the outlined handover capabilities, the RAPID5G Mobile IP system was designed with two significant additional features to further improve the quality of service in the multi-RAT environment. These two extensions are a FILS (Fast Initial Link Setup) based protocol and multi home support to connect to multiple RATs simultaneously.

FILS is a technology which was standardized by
IEEE802.11ai and reduces the initial link setup time [11]. The RAPID5G Mobile IP system incorporates a protocol based on FILS for the different RATs. Reducing the link setup time is especially relevant for high data rate and low latency services. For high data rate services, the initial link setup amounts to a substantial part of the time to e.g. download a file. For low latency services, a short link setup time is important to prevent failure in case the link was interrupted and must be reestablished.

In contrast to standardized Mobile IP implementations, the RAPID5G mobile IP system can associate multiple care-of addresses for multiple RATs to one home address. Thereby, the RAPID5G system is able to not only handover between RATs, but can also utilize several RATs at the same time. In addition to the potential to increase the overall throughput, different RAU can be employed for different applications, which run at the same time. Thus, applications can use the RAT, that suits their performance requirements best. This is especially relevant in the context of $5 \mathrm{G}$, where different services with vastly different performances with regards to battery consumption, latency, reliability and data rate are foreseen. Such a system could for example allow to run a mission critical application over one $5 \mathrm{G}$ service, without being affected by another application, that uses a high data rate service for video streaming.

\section{Field Trial Management Software and Diagnostic Panel}

For the purpose of the field trial, a custom-made management software was developed and installed on the RAPID5G mobile terminal. This software component made it possible to show a graphical representation of the key performance indicators of the tests to the broad audience gathered in the shopping mall. For instance, instantaneous throughput meter, round-trip delay time (RTD), video streaming quality and download time comparison widgets were displayed on the terminal's screen. In addition to the presentation layer for the audience, the management software also provided a diagnostic panel shown in Fig. 2. This panel allowed to monitor and to reconfigure every parameter of the system in real time. Testers were able to 
verify state and radio conditions of all three network interfaces $(5 \mathrm{G} 60 \mathrm{GHz}$, LTE and $\mathrm{WiFi})$ as well as the Mobile IP related configuration in a "user friendly way". Besides monitoring, the diagnostic panel provided a tool for quick re-configuration of automatic handover algorithm. With this tool it was possible to enhance the handover procedure by injecting simple scriptbased rules that were executed during the algorithm iteration. The flexibility of such an approach accelerated the preparation and tuning process during the field trial. Thanks to the modular buildup of the software stack remote configuration of e.g. the thresholds for the handover algorithm were possible. Thereby, a mobile operator could react to changes or problems in the network.

\section{RAPID5G PhySiCAl LAyER TECHNOLOGY AT A GLANCE}

RAPID5G aims at providing radio access technologies for 5G using an advanced photonic infrastructure for dense user environments. With this aim, RAPID5G directly targets the core of the eMBB use case family: high data rates for many users at the same time [1]-[2]. Therefore, optical fiber is employed to distribute and interconnect the different RAT technologies, as it provides sufficient bandwidth and wavelength division multiplexing techniques. In the following the key physical layer technology developed within RAPID5G are briefly introduced. This includes the DAS and the $60 \mathrm{GHz}$ $5 \mathrm{G}$ RAUs for the eMBB and the hotspot use case featuring beam switching and beam steering, respectively.

\section{A. Distributed Antenna System}

To interconnect the various RAUs in the shopping mall with the internet, a fiber-based distributed antenna system has been developed and implemented. The distribution network consists of a prototype version of the Corning ONE platform and Exatel's fiber infrastructure as shown in Fig. 1. The DAS platform spans from the integrated headend unit (IHU 1), physically located in Exatel's data center about $11 \mathrm{~km}$ apart from the shopping mall, to the IHU 2, located in the shopping mall and feeds multiple fiber connected LTE RAUs. Both IHU units are connected via dark fiber using Exatel's fiber network infrastructure. The LTE mobile service is fed into the radio interface module (RIM) of IHU 1 (input 1 in Fig. 1), where the signal is conditioned and amplified. For $2 \times 2$ MIMO operation two signal streams are fed into two different RIMs. The signals are provided by the mobile operator's LTE base station, consisting of a system module and a radio module. The RF signals are then modulated onto an optical carrier using amplitude modulation. This optical signal can be multiplexed with additional optical signals fed via input 2 in Fig. 1. This input is used in the field trial to provide the uncompressed optical video signals for the $5 \mathrm{G}$ hotspot.

The multiplexed optical signals are then transmitted via 11 $\mathrm{km}$ single mode fiber (SMF) to IHU 2. There the optical signals are de-multiplexed and the hotspot video signals are extracted (output 4 in Fig. 1). The optical LTE signal is converted into an electrical signal and reconditioned, before being reconverted into an optical signal by an optical interface module (OIM). After the OIM the optical LTE signal is transmitted to the interconnect unit (ICU), where it is coupled into a composite cable together with an optical Gigabit-Ethernet $(\mathrm{GbE})$ signal (output 5 in Fig. 1). The optical GbE signal is designated for the WiFi access point (AP) and the $60 \mathrm{GHz}$ RAU and converted from an electrical GbE signal, which is provided via input 3 in Fig. 1. The composite cable includes multiple optical fibers and power cables for the LTE RAUs and transports the optical signals to the to the LTE access point in the public area of the shopping mall. At the LTE RAUs an integrated Gigabit Ethernet module (GEM) extracts the GbE signals for the WiFi $\mathrm{AP}$ and the $60 \mathrm{GHz}$ transceivers.

\section{B. $5 \mathrm{G} 60 \mathrm{GHz}$ transceiver supporting $2 \mathrm{D}$ beam switching}

Utilizing mm-wave spectrum for cellular access has significant advantages in terms of spectrum availability and spectrum reuse capability [2],[6],[12]. Naturally, when using higher frequencies, more spectrum is available, so just by transitioning from operation at bands near $2 \mathrm{GHz}$ to a band around $60 \mathrm{GHz}$, a factor of 30 in the available spectrum resource may be gained. Furthermore, the size of the antenna elements at mm-wave frequencies becomes much smaller compared to frequencies below $5 \mathrm{GHz}$, thus enabling to deploy large arrays of antenna elements in a small space for higher order MIMO and smart beam-forming [2],[12].

The use of beam-forming has great potential for increasing the capacity of the system, as beams directed towards the intended recipient focus the radio energy in the desired direction, thus increasing the signal level on the intended recipient and decreasing the interference level at unintended recipients. The use of beam-forming enables transmitting different data to different recipients at the same time, thus practically enabling increasing the capacity of the network by the number of beams deployed in parallel. These advantages of mm-wave frequencies have been recognized by standardization bodies and regulators alike and have led to some mm-wave bands, at the moment primarily near $28 \mathrm{GHz}$, being an essential part of emerging 5G standardization [12].

Siklu has developed low-cost $60 \mathrm{GHz}$ transceiver chips and various beam forming technologies, including unique lensassisted beam switching antenna technology within the RAPID5G project. The SiGe technology based transceiver IC is highly integrated and includes all relevant elements from the baseband electronics, voltage controlled oscillator with a phase locked loop, frequency mixers and up to the RF TX and RX amplifiers on-chip. The beam switching antenna consists of a LTCC substrate integrating the radiating elements and an RFIC. The substrate can be used with a mm-wave lens or reflector to implement a beam-switching antenna, or without such passive focusing elements to implement a beam-forming antenna and is depicted in Fig. 3.

These technology components have been integrated together in a prototype (a model can be seen in Fig. 3) of a point-tomultipoint system that is capable of providing wireless connectivity using a $1 \mathrm{Gbps}$ copper Ethernet interface or a 2.5 Gbps SFP interface with over-the-air throughput of close to 2 Gbps in a TDD operation mode. The $60 \mathrm{GHz}$ RAU with 


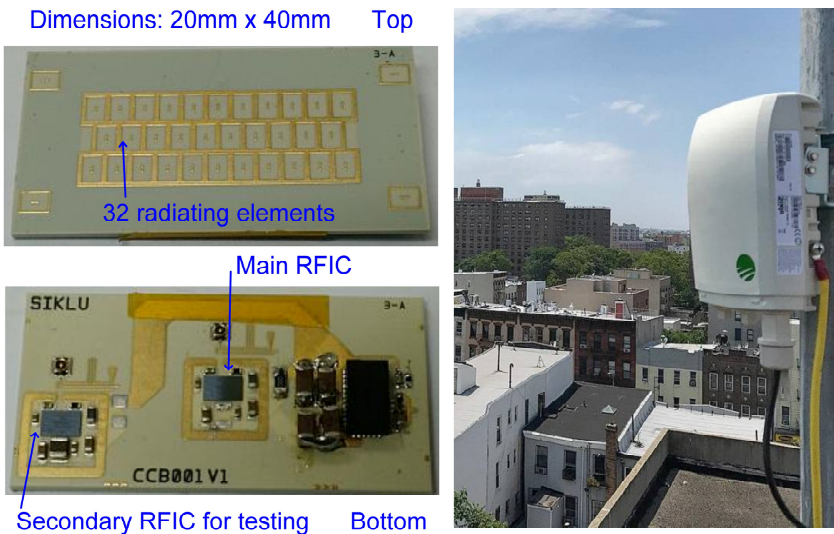

Fig. 3. LTCC integration of the radiating elements with the RFICs for the lensassisted beam switching antenna system for the $5 \mathrm{G} 60 \mathrm{GHz}$ transceiver (left) and a packaged $5 \mathrm{G} 60 \mathrm{GHz}$ transceiver in an outdoor installation (right).

integrated baseband modem, RFIC and lens assisted beam switching is utilized via the $1 \mathrm{Gbit} / \mathrm{s}$ copper interface in the field trial. It is supplied by Power-over-Ethernet (PoE), consuming between $6.9 \mathrm{~W}$ (idle) and $9.2 \mathrm{~W}$ (max. data rate). For high data rate and high reliability $500 \mathrm{MHz}$ bandwidth and up to 64QAM modulation with hardware integrated error correction coding is used [13]. The system has been deployed in the field trial area, whereby two $60 \mathrm{GHz}$ RAUs have been installed close to each other at the ceiling of the first floor to simulate closeby cells with no interferences observed. Further, a vertical handoff scenario between LTE, mm-wave and WiFi has been demonstrated. In this scenario, the mm-wave radio unit also performed a smooth horizontal handoff between the mm-wave RAUs. The $60 \mathrm{GHz} 5 \mathrm{G}$ RAUs are based on the WiGig standard and $5 \mathrm{G}$ functionality like beam switching has been implemented using MAC layer functionality, which can easily be adapted for other upcoming standards. The antenna and radio frontend were designed for operation at $60 \mathrm{GHz}$, but their design principles could be applied to other mm-wave bands that may be allocated for $5 \mathrm{G}$ systems.

\section{Hotspot $60 \mathrm{GHz}$ RoF RAU supporting $1 \mathrm{D}$ beam steering}

The $60 \mathrm{GHz}$ hotspot RAU developed by University Duisburg-Essen (UDE) was also installed at the ceiling of the shopping mall field trial area. A photo of the hotspot RAU is shown in Fig. 4. It contains a high frequency photodiode (PD), a $60 \mathrm{GHz}$ amplifier and a substrate-integrated waveguide leakywave antenna providing multiple simultaneously steerable beams in the $60 \mathrm{GHz}$ band. The electrical power consumption of the photonic hotspot RAU, dominated by the RF amplifier, was $3.95 \mathrm{~W}$. The data signals to be delivered to the mobile users via the $60 \mathrm{GHz}$ hotspot were each carrying $1.5 \mathrm{Gbit} / \mathrm{s}$ uncompressed video signals for real-time high resolution video streaming. Each video signal was first upconverted to a respective IF-subcarrier before being modulated onto the optical carrier, effectively yielding two optical baseband signals at different frequencies. After extraction at the shopping mall data room, the video signals are amplified and an optical local oscillator (LO) is added. The photodiode of the hotspot RAU generated the individual video signals in the $60 \mathrm{GHz}$ band by heterodyning the individual optical video signals with the optical LO, resulting in a baseband over fiber system with optical upconversion. Because each optical video signal has a different optical subcarrier frequency, the wireless video signals also have different RF carrier frequencies, all in the 60 $\mathrm{GHz}$ band. Thus, the highly directive leaky-wave antenna radiates the various video signals into different directions depending on their RF carrier frequencies. In other words, the leaky-wave antenna performs direct conversion from optical frequency division multiplexing to spatial multiplexing. This way, multiple users at different locations can be served simultaneously and the radio beams can be steered remotely by changing the optical IF frequency of the video signals [14].

As can be seen there are distinct differences between the two implemented $60 \mathrm{GHz} 5 \mathrm{G}$ RAUs. The RFIC based RAU uses optical or electrical baseband Ethernet as signal input, which is then remodulated with complex modulation and upconverted to $60 \mathrm{GHz}$. The photonic RAU uses optical IF or baseband input together with an optical LO for transparent upconversion. The RFIC based RAU is reconfigurable, integrated and complex and can work with different antenna solutions [13]. In contrast the photonic RAU is passive apart from the amplifier and provides beam steering based on the beat frequency of the optical inputs, making it simple and scalable.

\section{ShOPPING MALl Field TRIAL}

\section{A. Detailed field trial network architecture with optical backhaul and fronthaul}

In order to conduct the field trial with the multi-RAT wireless network in the public area of the shopping mall, a sophisticated network architecture was developed and installed. The network was built up using the RAPID5G physical layer technologies introduced above. The field trial was supported by a Polish mobile network operator.

In general, the shopping mall field trial can be divided into five main physical locations as illustrated in Fig. 5: the datacenter of the internet provider Exatel located in Perkuna (Exatel HQ), the datacenter of the mobile network operator, another datacenter of Exatel in Mory, the data room in the Blue City shopping mall, and the public location in the commercial

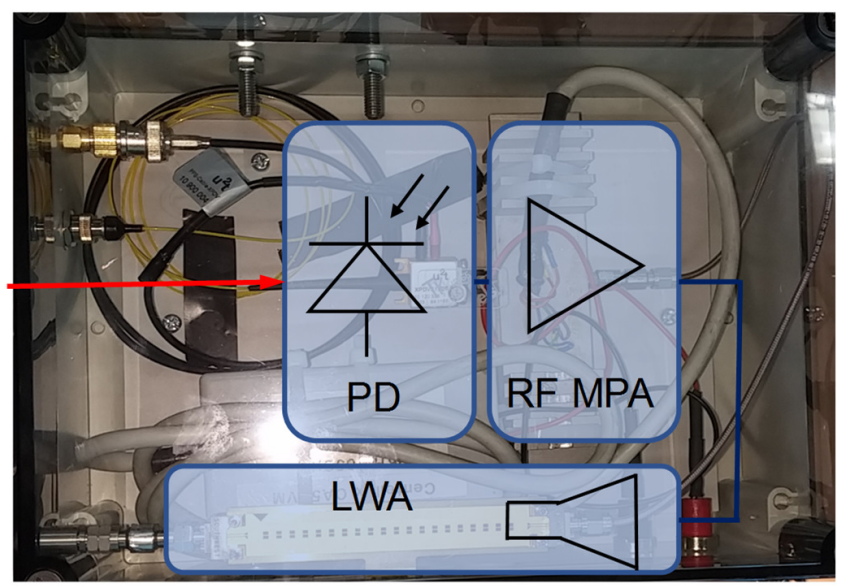

Fig. 4. Fabricated hybrid integrated RAU for the $5 \mathrm{G}$ hotspot ceiling installation consisting of a high-speed PD, a RF medium power amplifier (MPA) and a 60 $\mathrm{GHz}$ leaky-wave antenna (LWA). 


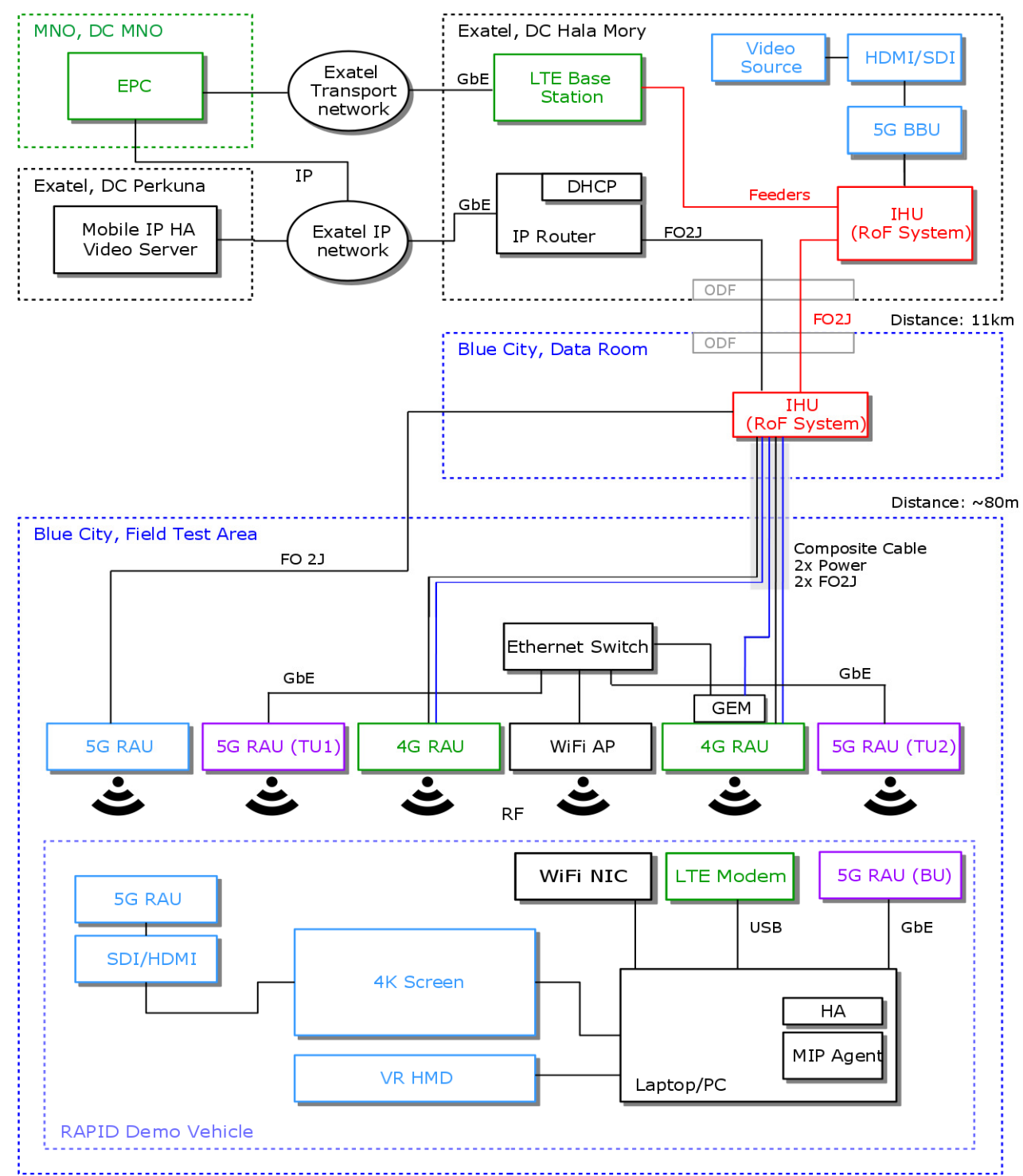

Fig. 5. Schematic architecture of the implemented network for the shopping mall field trial in Warsaw, Poland, with indicated physical locations. The color coding indicates the different radio access technologies (RATs). The abbreviations designate: Mobile IP HA: Mobile IP home agent, MNO: mobile network operator, DC: data center, GbE: Gigabit Ethernet, SDI: serial digital interface, BBU: baseband unit, DHCP: dynamic host configuration protocol, HA: handover agent, FO2J: fiber optic duplex cable, ODF: optical dark fiber, GEM: Gigabit Ethernet module, RAU: radio access unit, AP: access point NIC: network interface controller, TU: terminal unit, BU: base unit, VR HMD: virtual reality head mounted display, MIP: Mobile IP.

area of the shopping mall.

All signals $(60 \mathrm{GHz} 5 \mathrm{G}$ eMBB, $60 \mathrm{GHz} 5 \mathrm{G}$ hotspot, LTE, WiFi) used for the field trial are directly transferred to the shopping mall via the Exatel data center in Mory. The server cabinets in the Exatel's Mory DC are connected to a second server located in the Perkuna DC via Exatel's IP network. This second server provides the data accessed over LTE, WiFi or 60 $\mathrm{GHz} 5 \mathrm{G}$ eMBB service. The server also hosts the Mobile IP home agent. For supporting LTE in the shopping mall, a LTE base station was implemented in the Mory DC which is connected to the MNO's DC evolved packet core (EPC) using Exatel's GbE transport network. The DC in Mory was then connected via an $11 \mathrm{~km}$ long optical dark fiber (ODF) to the data room in the Blue City shopping mall. For the $5 \mathrm{G}$ eMBB service and $\mathrm{WiFi}$, the data was exchanged between Mory and the Blue City shopping mall using optical GbE.
From the Blue City data room all wireless signals are transmitted via optical fiber using the Corning DAS. Analog RoF [7] is used for fronthauling the UDE $60 \mathrm{GHz} 5 \mathrm{G}$ hotspot RAU as well as the LTE RAUs. The WiFi AP and the Siklu 5G eMBB RAUs were all connected by optical GbE backhaul via the IHUs in the Mory DC and the Blue City data room.

In the public field trial area, the WiFi AP, two LTE RAUs, two Siklu $60 \mathrm{GHz}$ eMBB RAUs and one UDE $60 \mathrm{GHz}$ hotspot RAU are all implemented at the celling and are all connected by the fiber-optic Corning DAS to the IHU in the Blue City data room. Composite cables were used to power the LTE RAUs and Ethernet cables were used on the last meter for providing PoE for the WiFi AP and the Siklu 5G $60 \mathrm{GHz}$ RAUs.

The RAPID5G mobile terminal demo vehicle developed by UDE and Exatel was equipped with adapters for all three RATs, i.e. WiFi, LTE and $60 \mathrm{GHz}$ 5G. Thus, the RAPID5G MT could 
connect wirelessly to each RAU and AP at the celling of the commercial shopping mall area. In addition to the MT, the demo vehicle was equipped with a computer hosting the Mobile IP agent, a $4 \mathrm{~K}$ video screen and a $4 \mathrm{~K}$ VR headset. The screen was accessed either by the computer or directly via the $5 \mathrm{G}$ hotspot receiver with a subsequent real-time SDI-to-HDMI converter.

\section{B. Public Demonstration}

In order to showcase seamless switching and seamless user experience enabled by Mobile IP and FILS for the eMBB use case, the RAPID5G demo vehicle is moved across the public area of the shopping mall while showing a cartoon video on its $4 \mathrm{~K}$ monitor. When the signal quality of the service over which the MT is connected drops below the set RSSI threshold or the MT is moved out of coverage, the implemented mobile IP home agent immediately switches to another available RAT. Therefore, the video stream on the MT is never interrupted. The video server employs the MPEG-DASH (dynamic adaptive streaming over HTTP) technique to stream and buffer the video in segments of $1 \mathrm{~s}$ duration and dynamically scale the quality according to the bandwidth of the used RAT. Additional demonstrations showed the throughput and sample file download time of the different wireless services live at different locations in the shopping mall. The performance in terms of signal quality and power, as well as upload/download throughput and round-trip delay time (RTD) is constantly analyzed and used for the handover algorithm. The diagnostic panel utilized for this is illustrated in Fig. 2. The logged download data rate and round trip delay from the field trial are plotted over a time index in Fig. 6. The active RATs are highlighted as well as the video streaming demonstration, where required data rate is limited and fluctuates. For the LTE service a maximum data rate of $47.1 \mathrm{Mbit} / \mathrm{s}$ was achieved under the real-life conditions in the shopping mall. Since we had used real LTE traffic from a Polish mobile operator, the handhelds of on average 200 shopping mall customers were automatically connected to our LTE base station. Consequently, our mobile terminal had to share the LTE cell capacity with those around 200 other users. In the laboratory, i.e. in a single user scenario, a maximum LTE data rate of $140 \mathrm{Mbit} / \mathrm{s}$ was achieved. The throughput of the $5 \mathrm{G}$ eMBB cells was up to $856 \mathrm{Mbit} / \mathrm{s}$, limited by the GbE interface. In the laboratory, we could achieve much higher cell capacities as we were not limited by the commercial GbE-interface, which was required for the field trial to interconnect all units and demonstrate RAT switching. Therefore, in the laboratory, cell capacities up to about $21 \mathrm{Gbit} / \mathrm{s}$ per polarization were achieved in the $60 \mathrm{GHz}$ band [15]. The latency between user and server corresponds to half the RTD, which amounted to minimum values of $2.2 \mathrm{~ms}$. So user latencies were mostly below $10 \mathrm{~ms}$ for the high data rate service and values close to $1 \mathrm{~ms}$ could be achieved. The quality of $5 \mathrm{G}$ service experience was also illustrated by a download speed comparison of a sample file of 35 Mbyte size. The 5G service showed consistent download times below $0.5 \mathrm{~s}$, which means that many cloud-stored files could be accessed as fast, as if they would be stored directly on the PC.

As initially indicated, RAPID5G targeted showcasing a high data rate multiuser hotspot in the field trial. The hotspot RAU can create multiple individually steerable beams as briefly described above. The number of beams is highly scalable, depending on the number of individual optical data channels. In the field trial, this was demonstrated by simultaneously transmitting in real-time two independent video channels in an

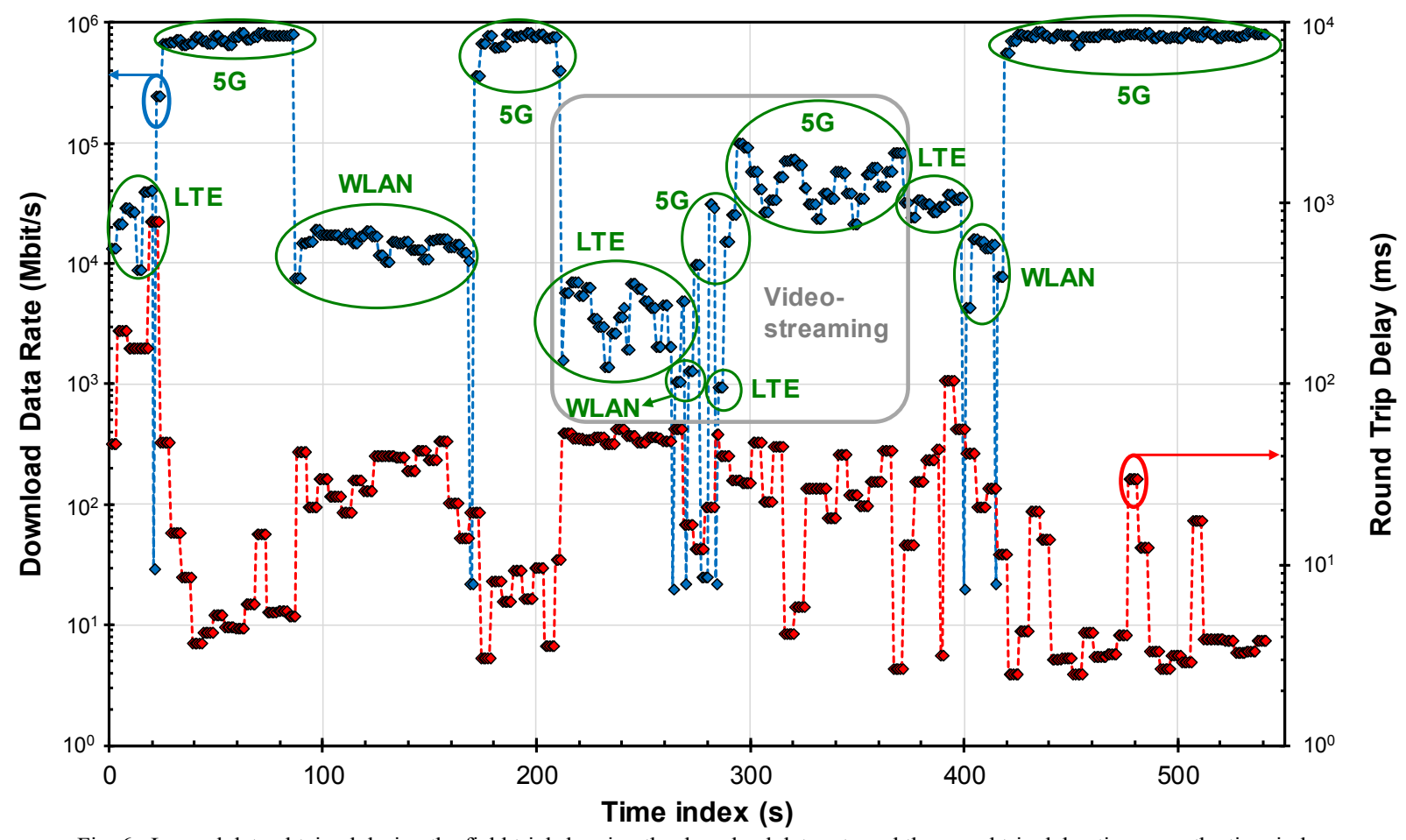

Fig. 6. Logged data obtained during the field trial showing the download data rate and the round trip delay time over the time index. 
uncompressed SDI (serial digital interface) format each requiring $1.5 \mathrm{Gbit} / \mathrm{s}$. In the hotspot those two video signals were radiated in the $60 \mathrm{GHz}$ band towards two different locations by the LWA. Since only one mobile terminal was available for the field trial, it had to be moved between the two locations to show the different videos. Of course, the hotspot could also be used to steer the beams to follow the mobile terminal. In the laboratory, we successfully connected multiple users to the same $5 \mathrm{G}$ hotspot from UDE [14]. Note that for receiving the $60 \mathrm{GHz}$ video signals, the mobile terminal was only equipped with a low-cost single diode Schottky-receiver for downconverting the video signals to baseband. After SDI to HDMI conversion, the real-time transmitted videos could be displayed using the $4 \mathrm{~K}$ monitor of the RAPID5G demo vehicle, which is shown in Fig. 1.

Finally, the RAPID5G network was also utilized to provide an $8 \mathrm{~K} 360^{\circ}$ video stream to a virtual reality (VR) headset to show the potential of a wireless VR experience. Wireless VR via $5 \mathrm{G}$ is specific use case that has great potential to drive the $5 \mathrm{G}$ market as it necessitates both, the low latency and the high data rates features of $5 \mathrm{G}$. The VR headset used in the field trial had a $4 \mathrm{~K}$ resolution, which was the highest resolution device available on the market at the time. It could be seen that the resolution was acceptable with some room for improvements and that for high quality $360^{\circ}$ video experience much larger resolutions than $8 \mathrm{~K}$ are required. Thus, it is likely that video streaming - which is already among the largest contributors to the overall mobile data traffic - will continue to rise further. In the field trial, the VR head tracking could be experienced by rotating point of view in the $360^{\circ}$ video via head movement. The data rate and latency of the $5 \mathrm{G} 60 \mathrm{GHz}$ service were sufficient for the VR application, which could be experienced by attending viewers via the video screen.

In summary, the following use cases were demonstrated in the RAPID5G shopping mall field trial:

1. Heterogeneous wireless access including $60 \mathrm{GHz}$ $5 \mathrm{G}, \mathrm{LTE}$ and WiFi

2. Distributed LTE coverage using a DAS and ARoF

3. Seamless vertical handover between $60 \mathrm{GHz} 5 \mathrm{G}$ cells and legacy WiFi and LTE wireless access via Mobile IP and FILS

4. Download rate and time comparison between 60 $\mathrm{GHz} 5 \mathrm{G}, \mathrm{LTE}$ and $\mathrm{WiFi}$

5. Two $60 \mathrm{GHz} 5 \mathrm{G}$ eMBB cells with $1 \mathrm{Gbit} / \mathrm{s}$ TDD service and automated $2 \mathrm{D}$ beam switching

6. Multiple user high data rate $60 \mathrm{GHz} 5 \mathrm{G}$ hotspot with $1.5 \mathrm{Gbit} / \mathrm{s}$ video streaming per channel

7. Virtual reality $8 \mathrm{~K} 360^{\circ}$ experience via $60 \mathrm{GHz} 5 \mathrm{G}$ service

\section{CONCLUSION}

We reported on a public field trial of a multi-RAT $(5 \mathrm{G}, 4 \mathrm{G}$, $\mathrm{WiFi}$ ) mobile network in the naturally dense user environment of a large shopping mall. We developed and implemented novel $60 \mathrm{GHz} 5 \mathrm{G}$ RAUs for the eMBB and hotspot use cases providing $2 \mathrm{D}$ beam switching and $1 \mathrm{D}$ beam steering, respectively. Within the shopping mall, all radio access points
(5G, LTE, WiFi) are connected to its data room via a fiber-optic distributed antenna system (DAS). The data room itself is interconnected to the data centers of an internet provider as well as to the data center of a mobile network operator via installed dark fiber. The $5 \mathrm{G}$ hotspot and LTE RAUs are fronthauled by analog RoF. The 5G eMBB RAU and the WiFi AP are backhauled via optical Gigabit Ethernet. By implementing a Mobile IP home agent and fast initial link setup (FILS) algorithm, we demonstrated seamless horizontal handover between $5 \mathrm{G}$ cells as well as vertical handover between $5 \mathrm{G}$, LTE and WiFi cells. Since the developed network provided commercial LTE service to the public, up to 200 customers in the shopping mall were connected to our network demonstrator. Technically, the latency of the $5 \mathrm{G}$ service was consistently below $10 \mathrm{~ms}$ of RTD with a minimum RTD of $2.2 \mathrm{~ms}$. The data rate within the $60 \mathrm{GHz} 5 \mathrm{G}$ eMBB was limited by the $\mathrm{GbE}$ interface connected to the novel integrated $60 \mathrm{GHz} \mathrm{SiGe}$ transceiver chip. The maximum data rate in the $5 \mathrm{G}$ eMBB cell was $856 \mathrm{Mbit} / \mathrm{s}$. By using the $60 \mathrm{GHz} 5 \mathrm{G}$ service, we demonstrated wireless virtual reality (VR) connectivity with an $8 \mathrm{~K} 360^{\circ}$ video that could be experienced by customers with a VR headset. The network was operational for over a week in the public space, highlighting the maturity of the developed technology.

\section{REFERENCES}

[1] D. Jiang and G. Liu, "An Overview of 5G Requirements," in 5G Mobile Communications, 1st ed., New York: Springer Publishing, 2016.

[2] R. J. Weiler et al., "Enabling 5G backhaul and access with millimeterwaves," 2014 European Conf. on Networks and Commun. (EuCNC), Bologna, 2014, pp. 1-5. doi: 10.1109/EuCNC.2014.6882644.

[3] Cisco Visual Networking Index: Global Mobile Data Traffic Forecast Update, 2016-2021. Mar. 2017. https://www.cisco.com/c/en/us/solutions/ collateral/service-provider/visual-networking-index-vni/mobile-whitepaper-c11-520862.html.

[4] Huawei, "5G opening up new business opportunities", Aug. 2017. http://www.huawei.com/en/xlabs/insights-whitepapers/5g-opening-upnew-business-opportunities-en

[5] K. Chandra, R. Venkatesha Prasad and I. Niemegeers, "An architectural framework for 5G indoor communications," 2015 Int. Wireless Commun. and Mobile Computing Conf. (IWCMC), Dubrovnik, 2015, pp. 11441149. doi: 10.1109/IWCMC.2015.7289244.

[6] J. Wells, "Faster than fiber: The future of multi-G/s wireless," in IEEE Microw. Mag., vol. 10, no. 3, pp. 104-112, May 2009. doi: 10.1109/MMM.2009.932081

[7] B. Lannoo et al., "Radio-over-fibre for ultra-small 5G cells," 2015 17th Int. Conf. Transparent Optical Networks (ICTON), Budapest, Hungary, 5-9 July 2015, doi: 10.1109/ICTON.2015.7193591.

[8] C. W. Chow, J. Y. Sung and C. H. Yeh, "A Convergent Wireline and Wireless Time-and-Wavelength-Division-Multiplexed Passive Optical Network," IEEE Photonics Journal, 2015, vol. 7, no. 3, pp. 1-7, doi: 10.1109/JPHOT.2015.2440339

[9] R. S. Koodli and C. E. Perkins, "Mobility Support Using Mobile IP," in Mobile Inter-networking with IPv6:Concepts, Principles and Practices, Wiley Telecom, doi: 10.1002/9780470126486.ch5

[10] J. M. Tilli and R. Kantola, "Data plane protocols and fragmentation for 5G," 2017 IEEE Conf. Standards for Commun. and Networking (CSCN), Helsinki, 2017, pp. 207-213. doi: 10.1109/CSCN.2017.8088623

[11] IEEE Approved Draft Standard for Information technologyTelecommunications and information exchange between systems-Local and metropolitan area networks-Specific requirements Part 11: Wireless LAN Medium Access Control (MAC) and Physical Layer (PHY) Specifications: Amendment to IEEE P802.11-REVmc(TM)/D8.0: Fast Initial Link Setup, in IEEE P802.11ai/D11.0 September 2016, pp.1-182, 2016.

[12] W. Roh et al., "Millimeter-wave beamforming as an enabling technology for $5 \mathrm{G}$ cellular communications: theoretical feasibility and prototype 
results" IEEE Commun. Mag., 2014, vol. 52, no. 2, pp. 106-113. doi: 10.1109/MCOM.2014.6736750.

[13] M. Steeg, M. Lange, Y. Leiba, A. Stöhr, Low-Latency GbE 60 GHz TDD Transceiver Using SiGe-RFICs and PCB Leaky-Wave Antennas, German Microwave Conference (Gemic 2018), Freiburg, 2018, pp. 156-159.

[14] M. Steeg and A. Stöhr, "High data rate $6 \mathrm{Gbit} / \mathrm{s}$ steerable multibeam 60 GHz antennas for 5G hot-spot use cases," 2017 IEEE Photonics Society Summer Topical Meeting Series (SUM), San Juan, 2017, pp. 141-142. doi: 10.1109/PHOSST.2017.8012690

[15] A. Stöhr, B. Shih, S. T. Abraha, A. G. Steffan and A. Ng'oma, "High spectral-efficient 512-QAM-OFDM $60 \mathrm{GHz}$ CRoF system using a coherent photonic mixer (CPX) and an RF envelope detector," 2016 Optical Fiber Commun. Conf. and Exhibition (OFC), Anaheim, CA, 2016, pp. 1-3

Matthias Steeg (Matthias.Steeg@uni-due.de) received his B.Sc. and M.Sc. degrees in electrical engineering and information technology from the University of Duisburg-Essen, Germany, in 2012 and 2015, respectively. After his studies with focus on micro- and optoelectronics he joined the Department of Optoelectronics, University of Duisburg-Essen, in 2015 as a Research Scientist. He is currently working toward his Ph.D. degree under the supervision of Prof. Stöhr. His research interests include radio-over-fiber techniques, mm-wave and $\mathrm{THz}$ communications, 5G mobile networks and mm-wave beam steering antennas.

Nathan J. Gomes (N.J.Gomes@kent.ac.uk) received his B.Sc. degree from the University of Sussex, United Kingdom, and his $\mathrm{Ph} . \mathrm{D}$. degree in electronic engineering from University College London, United Kingdom, in 1988. From 1988 to 1989, he held a Royal Society European Exchange fellowship with Telecom Paris, France. Since late 1989, he has been with the University of Kent, Canterbury, United Kingdom, where he is currently a professor of optical fiber communications. His current research interests include fiber-wireless access and radio-over-fiber systems and networks. He was the Technical Program Committee chair for the 2015 IEEE International Conference on Communications. He is a Senior Member of the IEEE.

Michał Kościesza received his M.Sc. degree in electrical and computer engineering from the Warsaw University of Technology, Poland in 2007. Since then, he has been working as a network architect and IT specialist for various mobile and fixed telecommunication operators. Currently he is a senior software engineer working for global mobile phones manufacturing company.

Adrian A. Juarez (juareza2@corning.com) received his Dipl.Ing. and Dr.-Ing degrees in electrical engineering from the Technical University of Berlin, Germany, in 2009 and 2015, respectively. Between 2007 and 2008 he was a research scholar at Duke University. From 2009 to 2014 he worked as a Research Scientist at Technical University of Berlin. In 2014 he joined Corning Optical Communications as an Optoelectronics Engineer.

Mason Lange (Mason.Lange@stud.uni-due.de) is studying electrical engineering and information technology at the University of Duisburg-Essen, Germany. Since 2014 he is working as a Research Assistant in the Department of Optoelectronics, University of Duisburg-Essen.
Yigal Leiba (Yigal@siklu.com) is a co-founder and CTO of Siklu. In previous positions, Yigal worked for Runcom Technologies, in charge of the design and the development of the world's first mobile-WiMAX (802.16e) chip, and a voting member of the IEEE 802.16 standard committee. Yigal was also a co-founder and CTO of Breezecom's LMDS group, a spin-off of Breezecom (later Alvarion (NASDAQ: ALVR)). His career includes project management and chief Engineer positions at Breezecom, where he was responsible for development of wireless access and wireless LAN systems. Before Breezecom, Yigal was an R\&D engineer in the Israeli Ministry of Defense Electronics Research Department. Yigal holds an MSc Degree in Electrical Engineering from the Technion - Haifa, Israel.

Hiroshi Mano (mano@koden-ti.com) established Root Inc. in 1993. Developed digital wireless communication devices and proposed a total network solution for convergence of analog and digital technologies. In addition, has been participating in numerous public and private councils and R\&D initiatives for WLAN-based high-speed mobile communications system development, technology enabling and commercialization, wireless adaptation and local information networking. A chair for IEEE 802.11 TGai WG for international standardization since 2010. And awarded Japan Communication Minister's Award 2017 for Information and Communication Technology Prize for the standardization efforts. In 2014, established EverySense, Inc. In U.S. Silicon Valley. EverySense developed an IoT information trading platform and acquired its national patent in Japan. Has been deeply involved in Japan and overseas in standardization and rule proposals in the fields of wireless communications, Internet, data trading, etc.

Hiroshi Murata (murata@elec.mie-u.ac.jp) received the B.Eng., M.Eng., and D.Eng. degrees in electrical engineering from Osaka University in 1988, 1990, and 1998, respectively, for studies on analyses on nonlinear-optic guided-wave systems and its applications to all-optical devices. In 1991, he joined the Department of Electrical Engineering, Faculty of Engineering Science, Osaka University, where he was an Associate Professor in Division of Advanced Electronics and Optical Science until the end of March 2018. In 2018, he joined the Graduate School Engineering, Mie University, Mie, Japan, where he is now a Professor. His works are concerned with electro optics, integrated optics, nonlinear optics and microwave-wave photonics. Dr. Murata is a member of the Institute of Electrical and Electronics Engineers (IEEE), The Optical Society (OSA), the European Microwave Association (EuMA), the Japan Society of Applied Physics, the Optical Society of Japan (OSJ), the Institute of Laser Engineering, and the Institute of Electronics, Information and Communication Engineers (IEICE).

Michał Szczęsny (Michal.Szczesny@exatel.pl) is Head of Architecture and Network Planning in Exatel. Formerly, in Polkomtel for many years responsible for managing of various architecture areas, from service solution architecture in network domain to enterprise architecture in IT domain. In professional activity mainly works on implementation of next generation networks and solutions - irrespective of the layer - from transport to service. An expert on the management of the 
technology strategy that is closely related to the expanding telecom market. He is an IEEE member and a voting member of IEC TC 103 and PKN TC 11.

Andreas Stöhr [SM’97] (Andreas.Stoehr@uni-due.de) received the Dipl.-Ing. and Dr.-Ing. degrees in electrical engineering from the Gerhard-Mercator-University, Germany, in 1991 and 1997, respectively. From 1987 to 1996 he was the CEO of MS Steuerungsanlagen GmbH in Germany. From 1996 to 2013, he was a Research Scientist at the University of Duisburg-Essen (UDE). During that period, in 1998 and 1999 he also joined the Communications Research Laboratory, Tokyo, Japan where he worked on $60 \mathrm{GHz}$ wireless systems employing radio over fiber techniques. He also worked with
France Telecom Orange Labs, Lannion, France, in 2009 and with Corning, Inc., in 2015. Since 2011, he is Professor and Head of the Optoelectronics Department, Center for Semiconductor Technology and Optoelectronics, UDE, Germany. His current research interests include III/V integrated microwave photonic devices and RF photonic integration for millimeter-wave and $\mathrm{THz}$ communications including $5 \mathrm{G}$, measurement systems as well as sensing applications. He has published more than 200 papers in refereed journals and conferences. He is a Senior Member of IEEE Photonics and MTT society, Committee Member and Chair of a number of international conferences and IEEE/OSA Guest Editor. 